\title{
Pick-up ions upstream and downstream of the termination shock
}

\author{
A. Czechowski ${ }^{1}$, H. J. Fahr ${ }^{2}$, G. Lay ${ }^{2}$, and M. Hilchenbach ${ }^{3}$ \\ 1 Space Research Centre, Polish Academy of Sciences, Bartycka 18A, 00-716 Warsaw, Poland \\ 2 Institut für Astrophysik und Extraterrestrische Forschung, Universität Bonn, Auf dem Hügel 71, 53121 Bonn, \\ Germany \\ 3 Max-Planck-Institut für Aeronomie, Max-Planck-Str. 2, 37191 Katlenburg-Lindau, Germany
}

Received 27 June 2001 / Accepted 6 September 2001

\begin{abstract}
Hydrogen pick-up ions produced in the inner heliosphere are convected outwards with the solar wind and suffer energy-diffusion by quasilinear wave-particle interactions. When arriving at the solar wind termination shock, the big majority of these pick-up's passes directly from the preshock to the postshock plasma regime. While crossing the shock these ions suffer a characteristic change in their density and velocity distribution which sensitively reflects the local shock properties. We extend the earlier model calculations to derive the pre-shock and postshock pick-up proton spectrum in the ecliptic plane, taking into account the global structure of the heliosphere and the termination shock. The proton distribution is used to calculate the energetic neutral hydrogen flux and the results are compared with the CELIAS/HSTOF data. There is a satisfactory agreement as regards the flux intensity scale and the directional dependence of the flux. The calculated energy spectrum is steeper than the data.
\end{abstract}

Key words. solar wind - acceleration of particles - cosmic rays

\section{Introductory remarks}

Since decades now the location and geometry of the solar wind termination shock has been a subject of intensive theoretical investigations (for a recent review see Zank 1999), though up to now no shock structure could yet be identified in direct or indirect signatures by the deepspace NASA spaceprobes. Meanwhile not only the location but even more the structure of the shock has become an object of prime interest since the multi-fluid character of this shock transition has clearly been recognized (Donohue \& Zank 1993; Zank et al. 1993; Chalov \& Fahr 1994, 1995, 1997; Le Roux \& Fichtner 1997; Kausch \& Fahr 1997; Fahr et al. 2000). For instance, dependent on the degree of the cosmic-ray-induced shock modulation and the pick-up ion (PUI) injection efficiency at the shock, density compression ratios between 1 and 6.5 can be expected. Furthermore not only will the shock be a non-classical one (i.e. classical Rankine-Hugoniot relations may not be applicable), it most likely also has the character of a weak shock with preshock solar wind Mach numbers much smaller than expected earlier (see Baranov \& Malama 1993; Fahr \& Rucinski 1999; Fahr et al. 2000). Contrary to earlier expectations (Potgieter \& Moraal 1988) the spectrum of anomalous cosmic rays originating near the shock is likely not to be of a power-law

Send offprint requests to: A. Czechowski, e-mail: ace@cbk.waw.pl type, not even at the shock position itself (see Le Roux \& Fichtner 1997; Dworsky \& Fahr 2000; Chalov \& Fahr 2000). For a better control of this open field of theoretical speculations by means of observational data directly relevant to these features we shall develop a theoretical description for post-shock pick-up ions in the heliospheric sheath region here and shall derive PUI-related energetic neutral atoms (ENA) fluxes and discuss their detectability at the position of the Earth.

\section{Calculation of upstream pick-up ion spectra}

Soon after their injection into the magnetized solar wind plasma flow, the pick-up ions isotropize their initial ring distribution due to effective pitch-angle scattering processes. The resulting pitch-angle isotropized distribution function, $f(t, \boldsymbol{r}, v)$, is found as a solution of the following kinetic transport equation (see e.g. Lee 1983; Isenberg 1987; Bogdan et al. 1991):

$$
\frac{\partial f}{\partial t}+\boldsymbol{U} \cdot \nabla f=\frac{1}{v^{2}} \frac{\partial}{\partial v}\left(v^{2} D \frac{\partial f}{\partial v}\right)+\frac{v}{3} \frac{\partial f}{\partial v} \nabla \cdot \boldsymbol{U}+S(\boldsymbol{r}, v)
$$

where $\boldsymbol{U}(\boldsymbol{r})$ is the solar wind velocity, $v$ is the velocity of pick-up ions in the solar wind rest frame, $D(\mathbf{r}, v)$ is the energy diffusion coefficient for pick-up ions, and $S(\boldsymbol{r}, v)$ is the local production rate of freshly ionized particles.

In the following we shall assume axial symmetry with the symmetry axis parallel to the LISM wind vector $\boldsymbol{V}_{\infty}$. 
This in effect restricts our considerations to the vicinity of the ecliptic plane. Using spherical polar coordinates $(r, \theta, \phi)$, with the Sun as the origin and the polar axis oriented to the interstellar upwind direction (i.e. $\theta=0=$ LISM upwind direction), the above transport equation (Eq. (1)) after introduction of the differential phase space density, $G=2 \pi r^{2} \sin \theta v^{2} f$ attains the following form (see Chalov et al. 1997):

$$
\begin{aligned}
\frac{\partial G}{\partial t}= & -\frac{\partial}{\partial r}\left(u_{r} G\right)-\frac{\partial}{\partial \theta}\left(\frac{u_{\theta}}{r} G\right)-\frac{\partial}{\partial v} \\
& \times\left[\left(\frac{\partial D}{\partial v}+\frac{2 D}{v} \frac{v}{3 r^{2}} \frac{\partial u_{r} r^{2}}{\partial r}-\frac{v}{3 r \sin \theta} \frac{\partial u_{\theta} \sin \theta}{\partial \theta}\right) G\right] \\
& +\frac{1}{2} \frac{\partial^{2}}{\partial v^{2}}(2 D G)+2 \pi r^{2} \sin \theta v^{2} S(r, \theta, v)
\end{aligned}
$$

where $u_{\mathrm{r}}=u_{\mathrm{r}}(r, \theta)$ and $u_{\theta}=u_{\theta}(r, \theta)$ are the radial and tangential components of the mass-loaded solar wind velocity. Equation (2) then is solved numerically by further converting this nonlinear partial differential equation of second order into an equivalent system of linear stochastic differential equations (SDE-system). Further details can be found in papers by Chalov et al. $(1995,1997)$ or by Fahr \& Lay (2000).

The injection source $S(r, \theta, v)$ is due to neutral $\mathrm{H}$ atoms which become ionized at $(r, \theta)$. These neutral LISM $\mathrm{H}$ atoms penetrate from interstellar space into the interface region of perturbed solar wind and interstellar plasma flows and with some depletion eventually appear in the inner heliosphere being subject to ionization processes there. When these neutral atoms are ionized, they constitute the $\mathrm{H}^{+}$-pick-up ion production rate $q(\boldsymbol{r})$. The local heliospheric pick-up $\mathrm{H}^{+}$-production rate is given by the following formula (see Rucinski et al. 1993):

$q(\boldsymbol{r})=n_{\mathrm{H}}(\boldsymbol{r})\left[\nu_{\mathrm{ph}}(\boldsymbol{r})+\nu_{\mathrm{ex}}(\boldsymbol{r})\right]$

where $n_{\mathrm{H}}(\mathbf{r})$ is the number density of the neutral H-atoms in the heliosphere, and $\nu_{\mathrm{ph}}(\boldsymbol{r})$ and $\nu_{\mathrm{ex}}(\boldsymbol{r})$ are the relevant local photoionization and charge exchange frequencies. In Chalov et al. $(1995,1997)$ the densities $n_{\mathrm{H}}(\boldsymbol{r})$ are calculated within the self-consistent twin-shock interface model developed by Baranov \& Malama (1993) with LISM parameters fixed in accordance with settings made by these authors. The total pick-up ion production rate given in Eq. (3) is related to the injection source $S(r, \theta, v)$ in Eq. (2) by

$q(r, \theta)=4 \pi \int v^{2} S(r, \theta, v) \mathrm{d} v$

We assume that $S \propto \delta(v-U)$, where $U$ is the local solar wind speed. Our assumption of a narrow initial pickup ion distribution is a simplification (see Zank \& Cairns 2000). The interplanetary magnetic field in reality will deviate from the Archimedean spiral as given by the Parker model and used here. The primary injection thus occurs into a plasma flow which has a varying magnetic field orientation, leading to different parallel component of the pick-up ions velocity. The freshly injected PUIs will be slipping with respect to the bulk flow and tend to accumulate in the regions where $B$ is perpendicular to the flow. This situation may be represented by an injection with a broadened delta peak. Also, as discussed by Zank \& Cairns (2000), the wave excitation during the pick-up in quasiperpendicular magnetic field is less efficient than expected. However, the effect on our results would be probably restricted to the low energy part of the spectrum, and the results for the energies significantly higher than the initial spectrum width would not be changed.

The pick-up ion spectra are obtained by numerical integration of the above mentioned SDE-system of linear differential equations and reflect the effects of convection, adiabatic cooling, and momentum diffusion. We use the phase-space pick-up ion distribution function $f^{\mathrm{PUI}}$ defined by $f^{\text {PUI }} \equiv \mathrm{d} N / \mathrm{d}^{3} x \mathrm{~d}^{3}\left(\boldsymbol{v} / U_{0}\right)$ where $\boldsymbol{v}$ is the particle velocity relative to plasma and $U_{0}=4.5 \times 10^{7} \mathrm{~cm} \mathrm{~s}^{-1}$ is the reference solar wind speed. The results from SDE calculations by Chalov, Fahr \& Izmodenov (Chalov et al. 1995) are expressed in terms of $\Phi^{\mathrm{PUI}} \equiv U_{0}\left[\mathrm{~d} N / \mathrm{d}^{3} x \mathrm{~d}\left(E / E_{0}\right)\right]$ $\left(E_{0}=m U_{0}^{2} / 2 \simeq 1 \mathrm{keV} / \mathrm{n}\right)$ which has the dimension of the differential flux (it is approximately equal to the pick-up ion differential flux integrated over the direction of particle velocity). $\Phi^{\mathrm{PUI}}$ is related to $f^{\mathrm{PUI}}$ by:

$f^{\mathrm{PUI}}=\frac{\Phi^{\mathrm{PUI}}}{2 \pi U_{0} \sqrt{w}}$

where $w$ denotes the normalized particle kinetic energy in the plasma frame: $w=\left(v / U_{0}\right)^{2}=\left(E / E_{0}\right)$.

In Fahr \& Lay (2000) the calculations were restricted to the upwind direction (i.e. $\theta=0$ ). The numerical results for $\Phi^{\mathrm{PUI}}(X, w)$ (where $X=\left(r / r_{\mathrm{E}}\right), r_{\mathrm{E}}=1 \mathrm{AU}$ ) in the pre-shock range $1 \leq X \leq X_{\mathrm{S}}(\theta=0)\left(X_{\mathrm{S}}(\theta) \equiv r_{\mathrm{S}}(\theta) / r_{\mathrm{E}}\right.$ is the normalized distance to the termination shock) can be parametrized in the form:

$\Phi^{\mathrm{PUI}}(X, w)=C_{\gamma}(X) w^{\gamma} \mathrm{e}^{-\left[C_{K}(X)\left(w-w_{0}\right)^{K}\right]}$

with $C_{\gamma}(X), C_{K}(X), K, \gamma$, and $w_{0}$ defined below. This corresponds to the following expression for the distribution function:

$f_{1}^{\mathrm{PUI}}(r, \theta=0, v)=\frac{C_{\gamma}(X) w^{\gamma} \mathrm{e}^{-\left[C_{K}(X)\left(w-w_{0}\right)^{K}\right]}}{2 \pi U_{0} \sqrt{w}}$.

Here the subscript "1" denotes the preshock spectrum.

We consider two different cases, corresponding to different behaviours of the Alfvenic turbulence with the distance from the Sun: $\left\langle\tilde{B}^{2}\right\rangle \propto r^{\alpha}$, where $\alpha=-3$ or -2 (Chalov et al. 1995). The first case $(\alpha=-3$, dissipationless wave propagation) is appropriate to solar minimum conditions (low turbulence). In this case the numerical results are best fitted by the following parameters

$$
\begin{aligned}
C_{\gamma}(X) & =10^{4.3141} X^{-0.3363}\left[\mathrm{~cm}^{-2} \mathrm{~s}^{-1} \mathrm{keV}^{-1}\right] \\
C_{K}(X) & =0.442 X^{0.202} \\
\gamma & =-0.1145 \quad K=(2 / 3) \quad w_{0}=0.8330
\end{aligned}
$$


The other case $(\alpha=-2)$ includes wave generation in interplanetary space and leads to the fit parameters:

$$
\begin{aligned}
C_{\gamma}(X) & =10^{4.1613} X^{-1.2567}\left[\mathrm{~cm}^{-2} \mathrm{~s}^{-1} \mathrm{keV}^{-1}\right] \\
C_{K}(X) & =0.469 X^{-0.5741} \\
\gamma & =-0.036 \quad K=(2 / 3) \quad w_{0}=9.7707 .
\end{aligned}
$$

It should be noted that while the first case $(\alpha=-3)$ corresponds to the average energy of the pick-up ions of the order of $2 \mathrm{keV}$ at large distance, so that the total energy in the pick-up ions is not larger than $0.3-0.5$ of the solar wind kinetic and thermal energy, the case $\alpha=-2$ is unrealistic: the average energy of the pick-up ion obtained from the resulting distribution function in this case may be as high as $100 \mathrm{keV}$. In this case too much energy is transferred from waves to the pick-up ions. Nevertheless, in the following we present some of the results obtained with $\alpha=-2$ using them as an illustration of an extreme case.

Our results are based on a simplified approach, in which the effect of wave driving by the PUIs was neglected. The studies of MHD turbulence behaviour in the outer heliosphere carried out by Zank et al. (1996) and Matthaeus et al. (1999), with the effects of mode mixing, driving by stream-stream interactions and wave excitation by interplanetary pick-up ions included in approximate form, produce the values $-10 / 4 \geq \alpha \leq-9 / 4$. To extend our approach to these cases would require including wavedriving in the transport equation from which the pick-up ions distribution function is derived.

Figures 1 and 2 present the evolution of the PUI distribution function with the distance in the upwind direction for these two cases together with the corresponding postshock distribution functions (see Sect. 3).

Our aim is now to generalize these results, derived for the upwind $(\theta=0)$ axis, to the general inecliptic regions with longitude angles $\theta \geq 0$. Instead of solving Eq. (2) for the non-spherically-symmetric case we shall assume that the distribution function can be derived from the solution for the upwind direction by a simple re-scaling with a direction dependent correction factor $\nu(\theta)$ :

$f_{1}^{\mathrm{PUI}}(r, \theta, w)=\nu(\theta) f_{1}^{\mathrm{PUI}}(r, \theta=0, w)$.

$\nu(\theta)$ is determined by requiring that the resulting distribution reproduces the directional dependence of the pickup ion number density at some reference distance. This approach requires the assumption that the interplanetary turbulence levels determining the momentum diffusion coefficients $D(\boldsymbol{r}, v)$ within the ecliptic only depend on the solar distance $r$, but not on longitude $\theta$. Momentum diffusion thus operates independent of longitude.

The correction factor $\nu(\theta)$ is given by

$\nu(\theta)=\frac{n^{\mathrm{PUI}}\left(r_{R}, \theta\right)}{\int f_{1}^{\mathrm{PUI}}\left(r_{\mathrm{R}}, \theta=0, v\right) \mathrm{d}^{3}\left(v / U_{0}\right)}$

where $r_{\mathrm{R}}$ is some reference distance (we take $r_{\mathrm{R}}=50 \mathrm{AU}$ ), $f_{1}^{\mathrm{PUI}}$ is given by Eq. (7), and $n^{\mathrm{PUI}}(r, \theta)$ is the pick-up ion

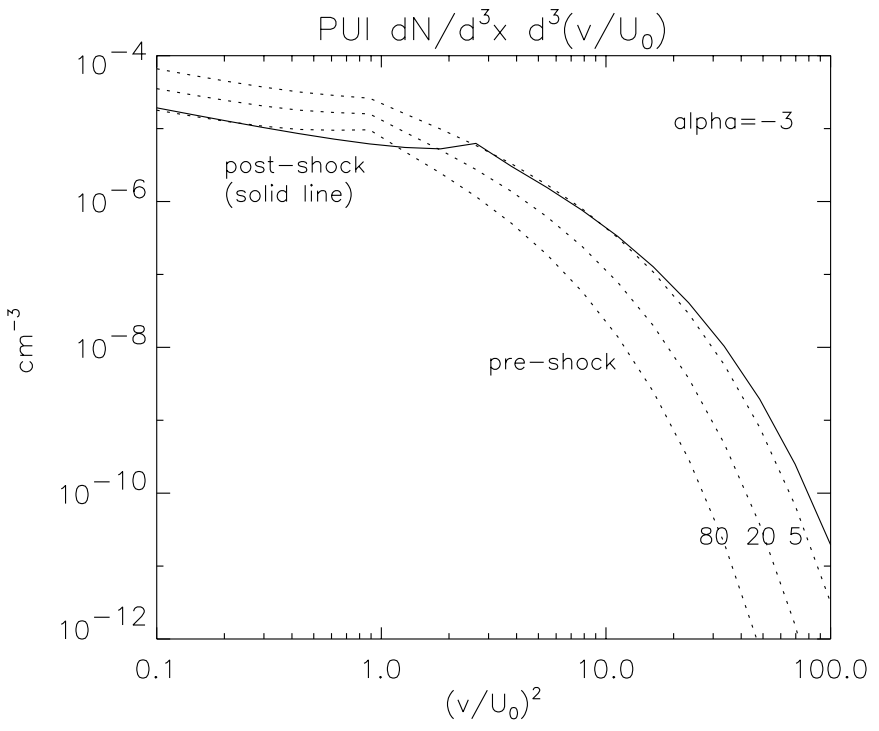

Fig. 1. PUI distribution function evolution with distance $(\alpha=$ -3 ). The functions for distances 5.0, 20.0 and 80.0 AU (apex direction) are presented by dotted lines. The distribution immediately downstream from the shock is shown by the solid line. (See Sect. 3).

number density at $(r, \theta)$ which we take from the model of the heliosphere based on a gas-dynamical 5-fluid numerical solution (Fahr et al. 2000) in which the pick-up protons were considered as one of the component fluids. The same model is used to provide the function $r_{\mathrm{S}}(\theta)$ defining the shape of the solar wind termination shock.

With the help of the above formulae one can give the near ecliptic PUI upstream spectra for longitudes $\theta \geq 0$ at the shock by

$$
\begin{aligned}
& f_{1}^{\mathrm{PUI}}\left(r_{\mathrm{S}}(\theta), \theta, v\right)= \\
& \quad \nu(\theta) \frac{C_{\gamma}\left(X_{\mathrm{S}}(\theta)\right) w^{\gamma} \mathrm{e}^{-\left[C_{K}\left(X_{\mathrm{S}}(\theta)\right)\left(w-w_{0}\right)^{K}\right]}}{2 \pi U_{0} w^{1 / 2}}
\end{aligned}
$$

where $X_{\mathrm{S}}(\theta)=r_{\mathrm{S}}(\theta) / r_{\mathrm{E}}, w=\left(v / U_{0}\right)^{2}$.

\section{Determination of postshock pick-up ion spectra}

We now want to derive the postshock PUI spectrum starting from the upstream spectrum at the shock given in Eq. (12). In the following we shall give expressions for spectra of those PUIs which upon arrival at $r=r_{\mathrm{S}}(\theta)$ directly pass over the local shock transition and consequently exclude those which undergo a reflection at the electric potential ramp of the shock and move upstream again (as described e.g. by Kucharek \& Scholer 1995; Chalov \& Fahr 1996, 2000; Lee et al. 1996; Zank et al. 1996; Giacalone \& Jokipii 1998; Scholer \& Kucharek 1999; Le Roux et al. 2000). The relative abundance of these reflected ions as calculated by Chalov \& Fahr (1996) is, however, of the order of 10 percent or less.

Here we use index " 1 " for upstream quantities and index " 2 " for corresponding downstream quantities. We assume that the distribution $f^{\mathrm{PUI}}$ is isotropic in velocity (i.e. due to strong pitch-angle diffusion by quasilinear 


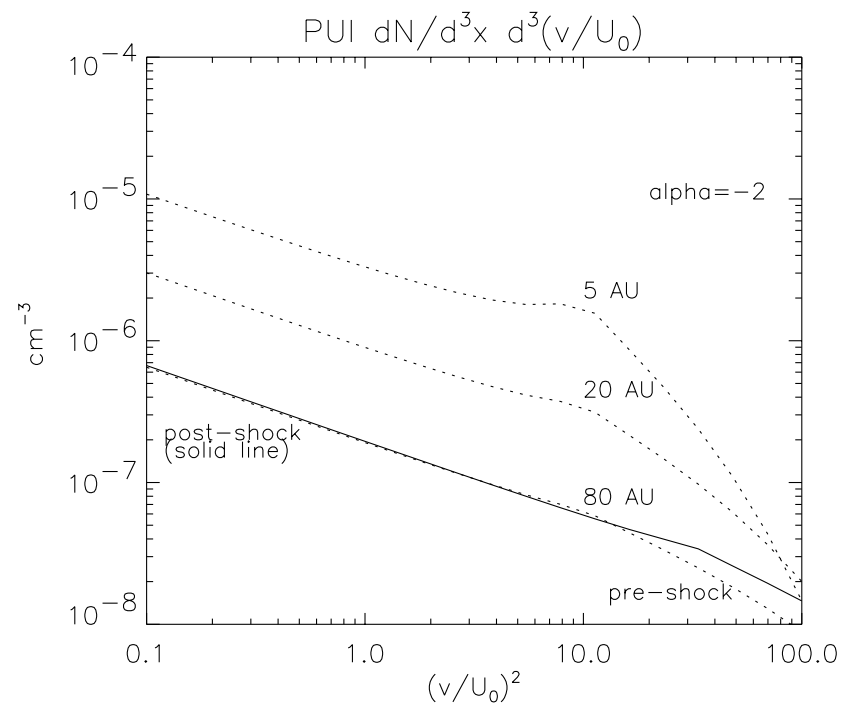

Fig. 2. PUI distribution function evolution with distance $(\alpha=$ -2 ). The functions for distances 5.0, 20.0 and 80.0 AU (apex direction) are presented by dotted lines. The distribution immediately downstream from the shock is shown by the solid line (see Sect. 3).

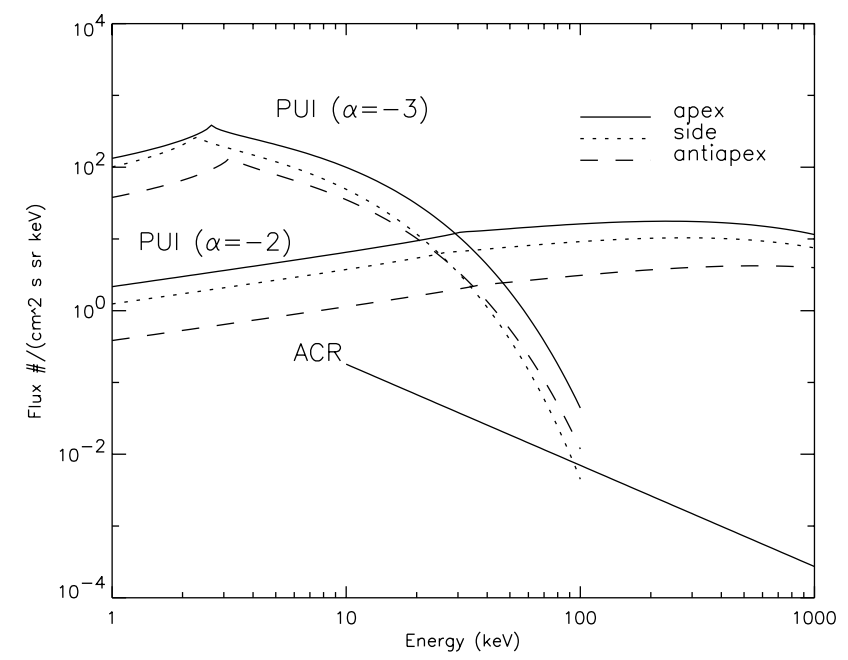

Fig. 3. Preaccelerated pick-up proton spectra immediately downstream from the termination shock (model predictions: Eq. (24)). The spectra are shown for three positions on the surface of the shock: $\theta=0^{\circ}$ (apex), 90 (side) and $180^{\circ}$ (antiapex) in the ecliptic plane. The cases of normal $(\alpha=-3)$ and high turbulence $(\alpha=-2)$ are presented. Also presented is the ACR spectrum at the shock derived from de-modulation of the observed spectrum (Stone et al. 1996).

wave-particle interaction). We also assume that the magnetic moment of the PUI is conserved when crossing the shock, which requires that the shock transition should be wide as compared to the PUI Larmor radius. At the PUI passage over the shock the following changes in the PUI distribution function have to be considered (we follow Fahr \& Lay 2000):

1) total densities are increased by the compression ratio:

$s(\theta)=\left[\rho_{2} / \rho_{1}\right]_{\theta}=\left[U_{1 \perp} / U_{2 \perp}\right]_{\theta}$ where $U_{1 \perp}$ and $U_{2} \perp$ are the upstream and downstream solar wind bulk velocity components perpendicular to the local shock surface.

2) due to the local downstream increase in the magnetic field magnitude by the local factor $\sigma(\theta)$ in connection with a conserved magnetic moment of PUIs passing over the shock there is a change in the particle energy characterized by:

$v_{\perp 2}^{2}=\sigma(\theta) v_{\perp 1}^{2}$

where $\boldsymbol{v}_{\perp}$ is the particle velocity perpendicular to the magnetic field. For an isotropic distribution $\left\langle v_{\perp}^{2}\right\rangle=(2 / 3) v^{2}$. This gives the result $v_{2}^{2}=\sigma(\theta) v_{1}^{2}$,

and:

3) due to the differential flux conservation over the shock (Liouville theorem) one obtains:

$f_{1}^{\mathrm{PUI}}\left(r_{\mathrm{S}}(\theta), \theta, v_{1}\right) \mathrm{d}^{3} v_{1} U_{1 \perp}=f_{2}^{\mathrm{PUI}}\left(r_{\mathrm{S}}(\theta), \theta, v_{2}\right) \mathrm{d}^{3} v_{2} U_{2 \perp}$

which leads to:

$$
\begin{gathered}
f_{2}^{\mathrm{PUI}}\left(r_{\mathrm{S}}(\theta), \theta, v\right)=f_{1}^{\mathrm{PUI}}\left(r_{\mathrm{S}}(\theta), \theta, v_{1}(v)\right) \frac{U_{1 \perp}}{U_{2 \perp}} \frac{\mathrm{d}^{3} v_{1}}{\mathrm{~d}^{3} v_{2}}= \\
s(\theta) J_{\theta}\left(v_{1}, v_{2}\right) f_{1}^{\mathrm{PUI}}\left(r_{\mathrm{S}}(\theta), \theta, v_{1}(v)\right)
\end{gathered}
$$

where $J_{\theta}\left(v_{1}, v_{2}\right)$ is the Jacobian of the transformation of preshock to postshock velocities given by: $J_{\theta}\left(v_{1}, v_{2}\right)=$ $\left.\left(4 \pi v_{1}^{2} \mathrm{~d} v_{1} / 4 \pi v_{2}^{2} \mathrm{~d} v_{2}\right)\right|_{\theta}=\sigma(\theta)^{-3 / 2}$ and where again $\sigma(\theta)$ is the field compression ratio at $r=r_{\mathrm{S}}(\theta)$ which is extracted from the result of the 5 -fluid counterflow simulation model published by Fahr et al. (2000) as we shall show below.

The derivation of $\sigma(\theta)$ is carried out by the following argumentation: at the position $r=r_{\mathrm{S}}(\theta)$ the upstream magnetic field can be split into components parallel and perpendicular to the shock surface, yielding:

$\boldsymbol{B}_{1}(\theta)=\boldsymbol{B}_{1 \|}+\boldsymbol{B}_{1 \perp}$

with

$B_{1 \|}=B_{1} \cos \xi \quad B_{1 \perp}=B_{1} \sin \xi$

with $\xi$ being the angle between the upstream field $\boldsymbol{B}_{1}$ and the shock surface (or between the upstream solar wind velocity $\boldsymbol{U}_{1}$ and the shock normal $\boldsymbol{n}(\theta)$ ). In the ecliptic the angle $\xi$ is connected with the local curvature of the shock surface and is given by:

$\operatorname{tg} \xi=r_{\mathrm{S}}(\theta) /\left[\partial r_{\mathrm{S}} / \partial \theta\right]_{\theta}=r_{\mathrm{S}} / r_{S, \theta}$

and is obtained from the form of $r_{\mathrm{S}}(\theta)$ given by Fahr et al. (2000). The downstream magnetic field now is given by:

$\boldsymbol{B}_{2}=\boldsymbol{B}_{2 \|}+\boldsymbol{B}_{2 \perp}$

with $B_{2 \|}=s(\theta) B_{1} \cos \xi$ and $B_{2 \perp}=B_{1} \sin \xi$.

The magnitude of the downstream magnetic field thus is given by:

$B_{2}(\theta)=s(\theta) B_{1}(\theta)\left[\cos ^{2} \xi+\sin ^{2} \xi / s^{2}(\theta)\right]^{1 / 2}$. 
Replacing now $\cos \xi$ and $\sin \xi$ by $\operatorname{tg} \xi$ given in Eq. (19) yields:

$\sigma(\theta)=s(\theta)\left(1+\operatorname{tg}^{2} \xi\right)^{-1 / 2}\left[1+\operatorname{tg}^{2} \xi / s^{2}(\theta)\right]^{1 / 2}$.

Taking all these results together leads to:

$f_{2}^{\mathrm{PUI}}\left(r_{\mathrm{S}}(\theta), \theta, v\right)=s(\theta) \sigma(\theta)^{-3 / 2} f_{1}^{\mathrm{PUI}}\left(r_{\mathrm{S}}(\theta), \theta, \sigma^{-1 / 2} v\right)$.

This on basis of Eq. (12) delivers the following postshock PUI spectrum:

$$
\begin{aligned}
& f_{2}^{\mathrm{PUI}}\left(r_{\mathrm{S}}(\theta), \theta, v\right)=\nu(\theta) s(\theta) \sigma(\theta)^{-3 / 2} \\
& \quad \times \frac{C_{\gamma}\left(X_{\mathrm{S}}(\theta)\right) \sigma(\theta)^{-\gamma} w^{\gamma} \mathrm{e}^{-\left[C_{K}\left(X_{\mathrm{S}}(\theta)\right) \sigma(\theta)^{-K}\left(w-w_{0}\right)^{K}\right]}}{2 \pi U_{0} \sqrt{w}}
\end{aligned}
$$

with $X_{\mathrm{S}}(\theta)=r_{\mathrm{S}}(\theta) / r_{\mathrm{E}}$ and $w=\left(v / U_{0}\right)^{2}$.

The resulting PUI fluxes immediately downstream of the shock are presented in Fig. 3 for three different positions at the shock surface. These are compared with extrapolated anomalous cosmic ray proton spectrum at the shock deduced from de-modulation of the observed highenergy ACR spectrum (Stone et al. 1996).

\section{Pick-up protons beyond the termination shock}

In the region downstream from the termination shock the plasma flow is slower $\left(\sim 100 \mathrm{~km} \mathrm{~s}^{-1}\right)$ and the temperature higher $(\sim 150-300 \mathrm{eV})$ than in the region upstream. The protons picked up by the flow will then be mixed with the thermal population.

To estimate the post-shock spatial and energetic distributions of the pick-up ions originating in the upstream region we apply the method used previously in the study of the low-energy ACR distribution (see Czechowski et al. 1995, 1999c, 2001; an application to PUI was presented in Czechowski et al. 1999a). The pitch-angle-averaged distribution function is obtained as a solution of Parker's transport equation. The PUI distribution function immediately after the shock obtained in the previous section is used as a boundary condition for the numerical solution downstream.

The solution downstream will thus be shaped by the effects of convection by the plasma flow (described by the velocity field $\boldsymbol{U}(\boldsymbol{x})$ ), losses predominantly due to chargeexchange with the neutral background atoms (loss rate $\beta$ ), spatial diffusion described by the diffusion tensor $\kappa$ and adiabatic energy change due to nonzero divergence of plasma flow $\nabla \cdot \boldsymbol{U}$. The plasma flow and its divergence (and also the background neutral density needed to determine $\beta$ ) are taken from Kausch's model (Kausch 1998; Fahr et al. 2000). The charge-exchange cross sections are taken from Barnett et al. (1990). We make the scalar diffusion approximation, which in the region between the termination shock and the heliopause may be adequate in lowest order. The diffusion coefficient is assumed to change at the heliopause. Inside the heliopause we assume $\kappa=\kappa_{1}=(1 / 3) \kappa_{\|}$with $\kappa_{\|}$the parallel diffusion coefficient derived from the modulation models (we take the formula of Le Roux et al. 1996) while outside the heliopause the

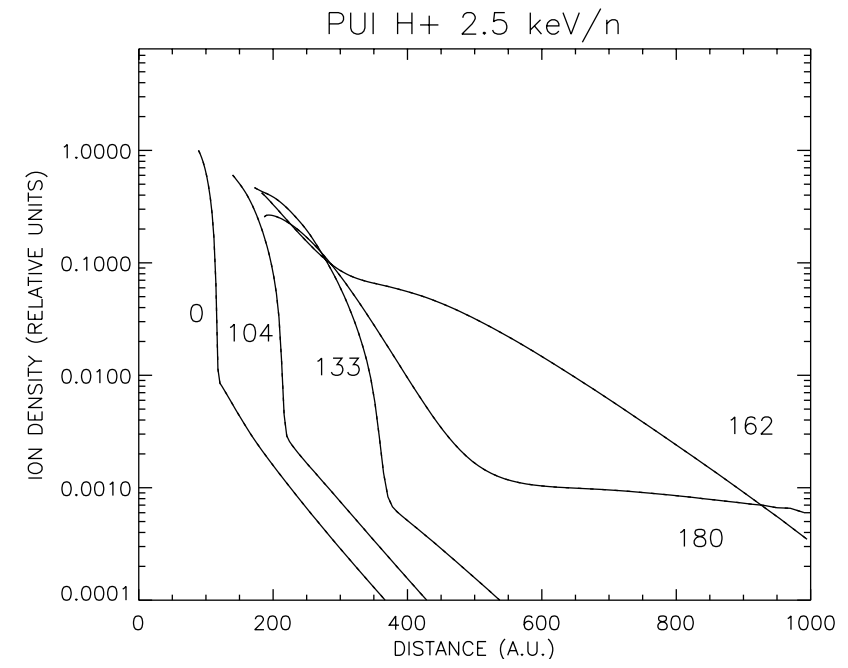

Fig. 4. PUI density distribution for $2.5 \mathrm{keV}$ protons outside the termination shock. The density profiles are shown for $\theta=0^{\circ}$, $104^{\circ}, 133^{\circ}, 162^{\circ}$ and $180^{\circ}$.

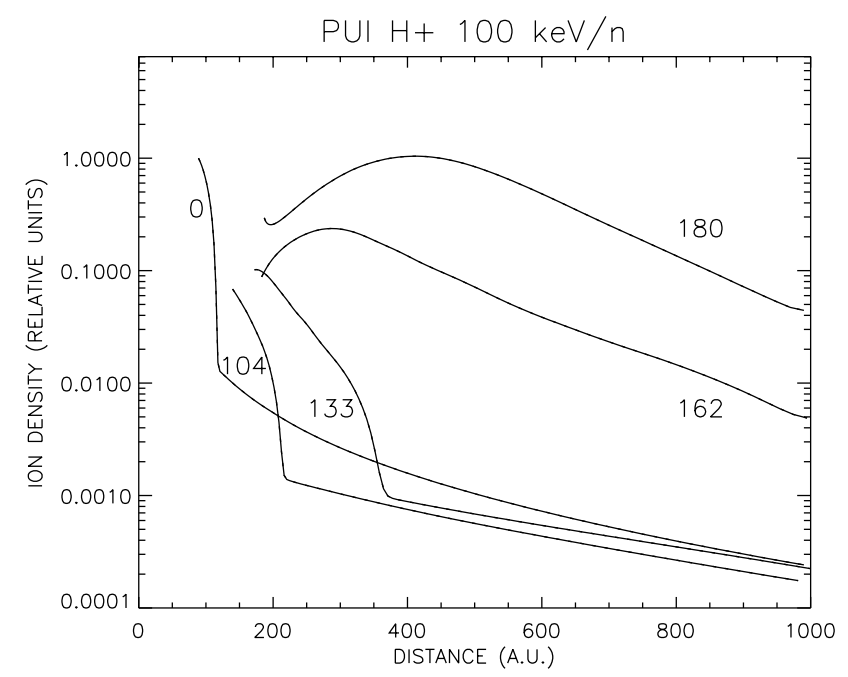

Fig. 5. PUI density distribution for $100 \mathrm{keV}$ protons outside the termination shock. Note the proton density enhancements due to adiabatic acceleration caused by compression of the flow in the heliotail region.

diffusion coefficient is taken to be much larger than inside: $\kappa_{2}=10^{2} \kappa_{1}$.

Parker's equation for the pitch angle averaged distribution function $f$

$$
\frac{\partial f}{\partial t}=\nabla \cdot \kappa \cdot \nabla f-\boldsymbol{U}(\boldsymbol{x}) \cdot \nabla f+\frac{1}{3} \frac{\partial f}{\partial \log p} \nabla \cdot \boldsymbol{U}-\beta f
$$

(the drift speed term is omitted, because it is small in the low energy range we are interested in). The equation was derived (Parker 1965) for the case of particles moving much faster than the background plasma. Downstream of the shock the plasma flows at the speed of less than $100 \mathrm{kms}^{-1}$. We take the low energy cutoff at $1 \mathrm{keV}$ corresponding to the particle speed of $\approx 450 \mathrm{~km} \mathrm{~s}^{-1}$. 


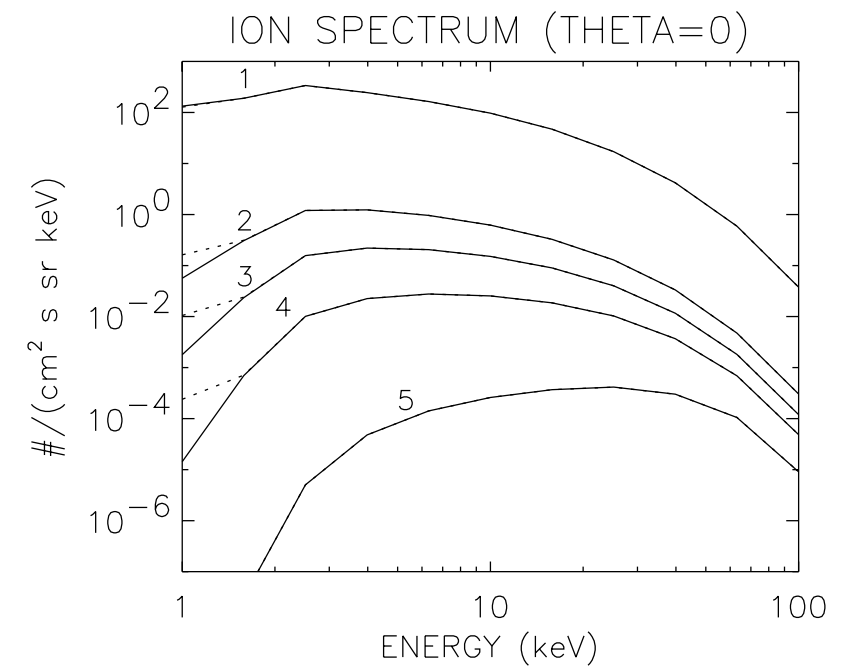

Fig. 6. Evolution of the PUI energy spectrum with heliocentric distance downstream from the shock (apex direction). The curves labelled 1 to 5 correspond to the distances of $89 \mathrm{AU}$ ( shock), 159 AU, 271 AU, $446 \mathrm{AU}$ and 990 AU, respectively. The solid (dotted) lines illustrate the effect of different choices of the lower boundary value in energy (at which the constant slope of the spectrum was assumed): $0.63 \mathrm{keV}(1 \mathrm{keV})$.

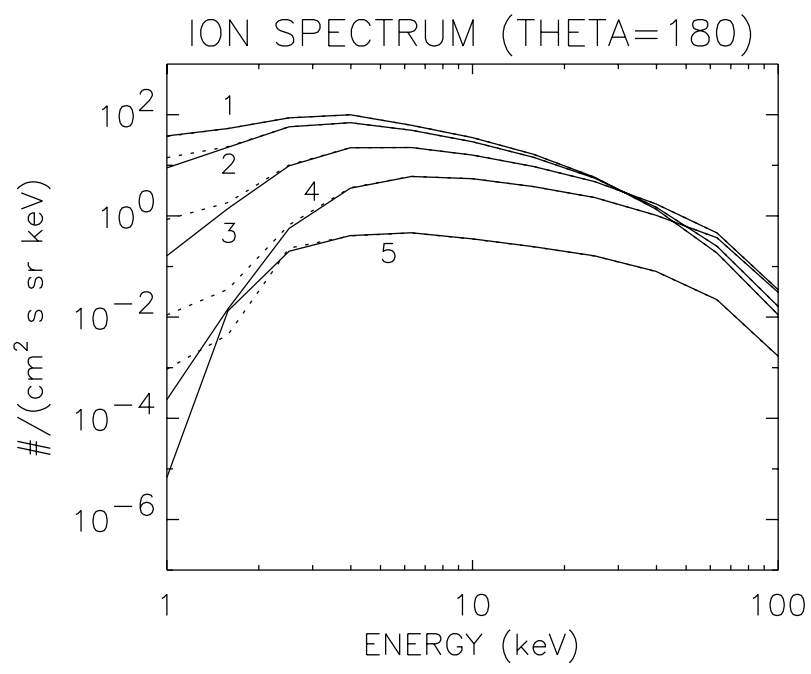

Fig. 7. As Fig. 6, but in the anti-apex direction. The curves labelled 1 to 5 correspond to the distances of $187 \mathrm{AU}$ ( $\sim$ shock $)$, $249 \mathrm{AU}, 345 \mathrm{AU}, 505 \mathrm{AU}$ and $990 \mathrm{AU}$, respectively. Note the crossing of the curves, related to the PUI density enhancements in the heliotail (Fig. 5).

We assume the solution to be axially symmetric with respect to the LISM flow apex-antiapex line. As the arguments leading to the post-shock form of the distribution are applicable only in the ecliptic, we must interpret the result as applicable also only near to the ecliptic plane.

The boundary conditions used in our approach are defined as follows. At the termination shock surface we set the distribution function $f(\boldsymbol{x}, v)$ to be equal to the postshock form of the upstream solution. At large distance the solution is required to match the asymptotic form (corresponding to constant plasma speed $\left.\boldsymbol{U}(\boldsymbol{x})=\boldsymbol{V}_{\infty}\right)$. At the boundaries in energy space we prescribe the slope of the energy spectrum, which we set to the value of the slope of the downstream shock spectrum.

The choice of the boundary conditions in energy, which must be done at each point in space, is (except for the requirement of consistency with the energy spectrum assumed at the shock) an arbitrary procedure, unless the behaviour of the ion distribution at the limits in energy is known (Czechowski et al. 1999c, 2001). To estimate the resulting uncertainty we have checked different cases, varying the energy limits and assuming different slopes. We found that the choice of the boundary conditions in energy affects the solution in the region close to the energy limits, but the effect in the remaining region is small.

The calculations were done for two cases, corresponding to two cases of the upstream solution: $\alpha=-3$ and $\alpha=-2$ (the latter case is the extreme one used only for comparison, see Sect. 1). Because the proton flux behaves differently for these cases, we set the upper energy limit to $100 \mathrm{keV}$ for the first case and to $1000 \mathrm{keV}$ for the second. The low energy limit we set to $1 \mathrm{keV}$ (see above).

The spatial pick-up protons distributions downstream from the shock for two values of energy are presented in Figs. 4 and 5 in the form of constant direction profiles. The angle $\theta$ is counted from the LISM apex direction. The density profiles start at the termination shock (which has the radius dependent on $\theta$ i.e. is non-spherical) and show a sharp change in slope at the heliopause. In Fig. 4 one can see a trough in the low energy $(2.5 \mathrm{keV})$ proton density near $\theta=180^{\circ}$, which is responsible for the dip in the low energy $(<3 \mathrm{keV})$ ENA flux from the antiapex direction (see Fig. 9). Figure 5 presents the typical distribution in the high PUI energy range $(100 \mathrm{keV})$. Because the PUI energy spectrum is steep at high energy the effect of the adiabatic acceleration in the heliotail (in the region where $\nabla \cdot \boldsymbol{U}<0$ : Czechowski et al. 2001) is quite pronounced (a "bump" in the $\theta=162^{\circ}$ and $180^{\circ}$ profiles in Fig. 5).

The energy spectra are presented in Figs. 6 and 7 for different heliocentric distances. The higher depletion of low energy particles with the distance is caused by chargeexchange processes (Czechowski et al. 1999c).

\section{Energetic neutral atoms from the pick-up ions}

The post-shock PUI distribution cannot as yet be observed in situ. One possibility is an indirect observation using as messengers the energetic neutral atoms (ENA), into which the PUI are converted by capturing electrons from the atoms of the background at the occasion of chargeexchange collisions (the first discussion of the heliospheric ENA was given by Hsieh et al. 1992). Below we calculate the flux of ENA corresponding to the PUI distributions derived above.

The flux of ENAs observed at the point $\boldsymbol{x}_{0}$ from the direction $\boldsymbol{n}: j_{\mathrm{ENA}}\left(\boldsymbol{x}_{0}, \boldsymbol{n}, E\right)$, can be obtained integrating along the line-of-sight $\boldsymbol{x}_{s}=\boldsymbol{x}_{0}+\boldsymbol{s} \boldsymbol{n}$ the flux of pickup ions relative to the neutral component multiplied by the density of the background neutral atoms and by the 


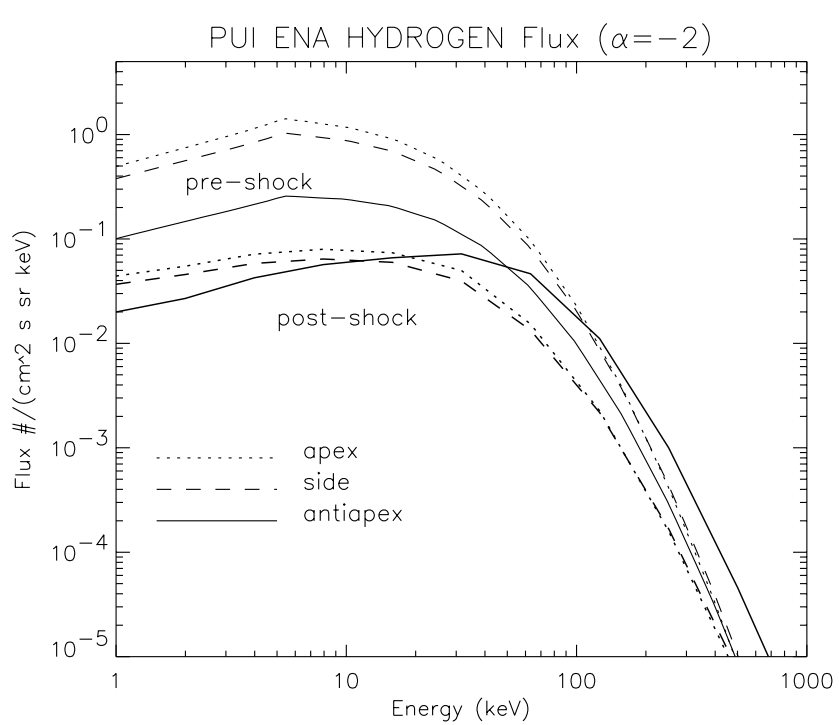

Fig. 8. PUI-ENA H spectra $(\alpha=-2)$ from outside and from inside of the termination shock. Three directions of the ENA flux are shown: from the LISM apex, from the direction at right angle to it in the ecliptic plane, and from the anti-apex direction. The post-shock spectrum is shown by the thicker lines.

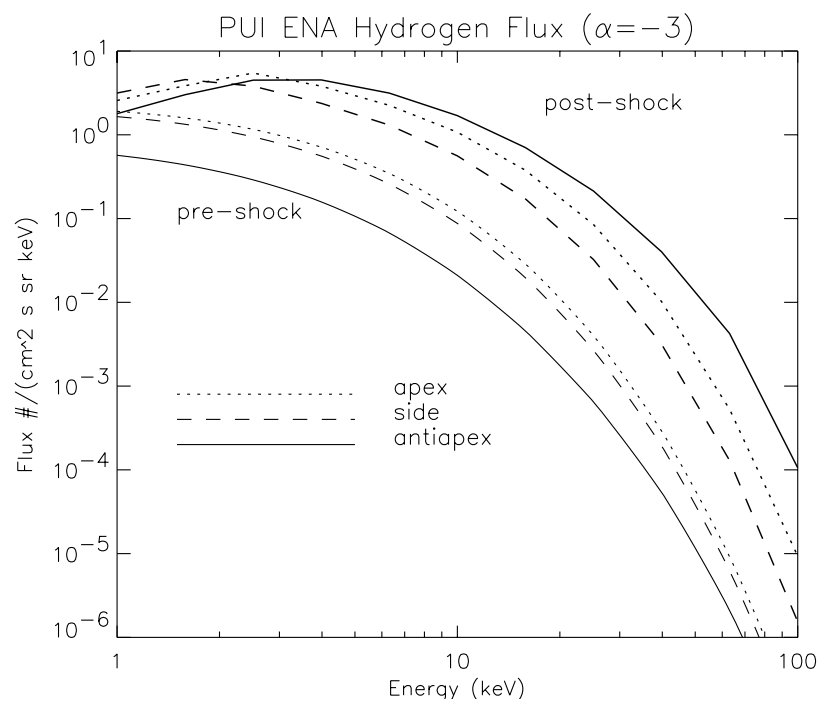

Fig. 9. PUI-ENA H spectra $(\alpha=-3)$ from outside and from inside of the termination shock. Three directions of the ENA flux are shown: from the LISM apex, from the direction at right angle to it in the ecliptic plane, and from the anti-apex direction. The post-shock spectrum is shown by the thicker lines.

charge-exchange cross section. The neutral components move at low speed in the fixed frame, so that we can use as a good approximation the PUI flux in the fixed frame: $j_{\text {PUI }}$ and neglect the difference between the velocity relative to the observer and that relative to the neutral background:

$$
\begin{aligned}
j_{\mathrm{ENA}}\left(\boldsymbol{x}_{0}, \boldsymbol{n}, E\right) & =\int_{0}^{\infty} \mathrm{d} s j_{\mathrm{PUI}}\left(\boldsymbol{x}_{s}, \boldsymbol{n}, E\right)\left[n_{\mathrm{H}}\left(\boldsymbol{x}_{s}\right) \sigma_{\mathrm{cx}, \mathrm{H}}(E)\right. \\
& \left.+n_{\mathrm{He}}\left(\boldsymbol{x}_{s}\right) \sigma_{\mathrm{cx}, \mathrm{He}}(E)\right] \operatorname{ext}\left(\boldsymbol{x}_{s}, E\right)
\end{aligned}
$$

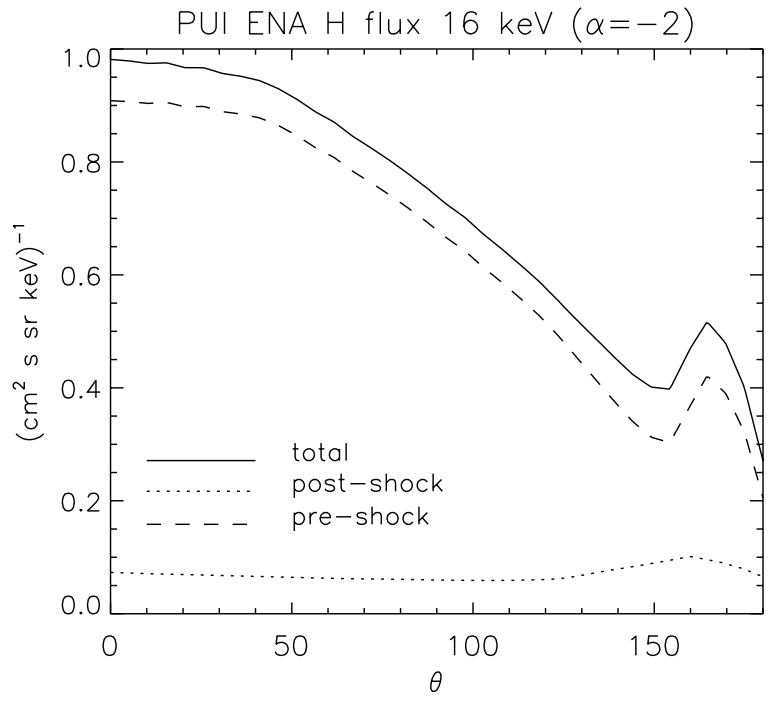

Fig. 10. PUI ENA $\mathrm{H}$ flux as a function of direction for $16 \mathrm{keV}$ $\mathrm{H}(\alpha=-2$ case). The post-shock and pre-shock contributions are shown by the dashed lines. The angle $\theta$ is counted from the apex direction.

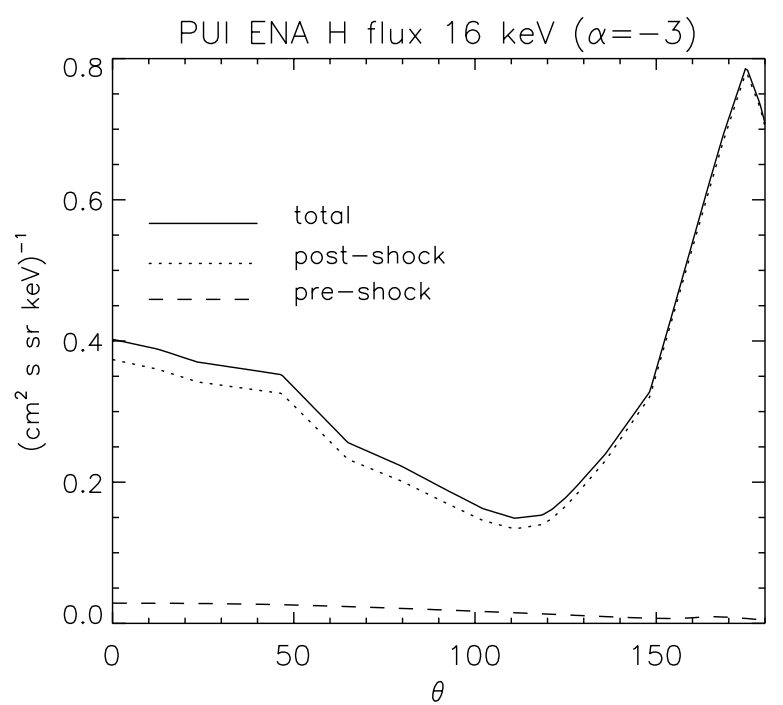

Fig. 11. PUI ENA $\mathrm{H}$ flux as a function of direction for $16 \mathrm{keV}$ $\mathrm{H}(\alpha=-3$ case $)$. The post-shock and pre-shock contributions are shown by the dashed lines. The angle $\theta$ is counted from the apex direction.

where $\operatorname{ext}\left(\boldsymbol{x}_{s}, E\right)$ is the extinction factor describing the losses of the second generation by charge-exchange and stripping.

The PUI ENA hydrogen flux corresponding to the model pick-up proton distributions is presented in Figs. 8-12. The calculations were done for the case of observations from the inner solar system (e.g. Earth's orbit) with the line-of-sight directed radially away from the Sun. Figures 8 and 9 show the PUI ENA spectrum for the cases $\alpha=-2$ and $\alpha=-3$, respectively. Note that the pre-shock ENA flux is negligible compared to the post-shock contribution in the normal turbulence $(\alpha=-3)$ case. This is obviously due in part to the effect of plasma motion (if the 


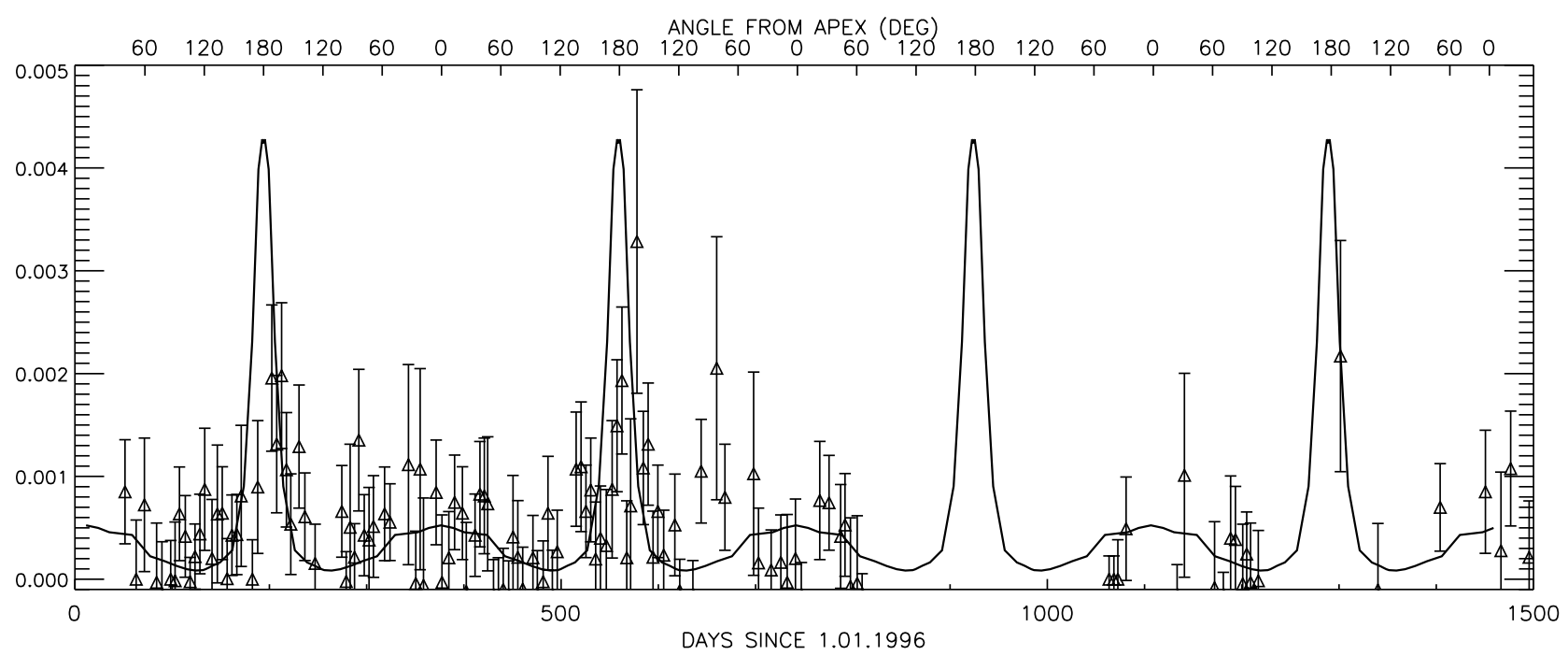

Fig. 12. ENA flux: the data points show the flux (units $\left.\left(\mathrm{cm}^{2} \mathrm{~s} \mathrm{sr} \mathrm{keV}\right)^{-1}\right)$ of mass $=1$ particles in low energy channels $(\sim 58-$ $88 \mathrm{keV}$ ) observed during quiet times (low ion flux) by CELIAS/HSTOF instrument. The results are the revised ones, taking the cross-calibration with ACE and IMP8 into account (Hilchenbach et al. 2000). Superimposed curve represents the calculated flux in the field of view of the instrument, of $63 \mathrm{keV}$ hydrogen ENAs from PUI transcharging for the case $\alpha=-3$ (the flux for $\alpha=-2$ case is too high by an order of magnitude).

pre-shock PUI velocity distribution in the plasma frame would be cut off at the solar wind energy there would be no sunward ENA flux at all). In the $\alpha=-2$ case the result is opposite due to strong pre-acceleration: this case is, however, unrealistic.

Figures 10, 11 illustrate the directional dependence of the ENA flux for the sample energy value of $16 \mathrm{keV}$ (the flux for $63 \mathrm{keV}$ for the case $\alpha=-3$ is shown in Fig. 12). In the normal turbulence case $(\alpha=-3)$ there is a pronounced peak in the flux from the anti-apex direction, provided the energy is not too low (see also Fig. 9). The peak is due to the concentration of the PUI in the heliotail (Figs. 4 and 5) due to convection by the plasma flow and to the asymmetric shape of the heliopause. The position of the minimum of the flux is away from the apex. The (unrealistic) $\alpha=-2$ case, where the flux is dominated by the contribution from the pre-shock region, behaves differently, with the maximum flux coming from the apex direction.

\subsection{Comparison with observations}

The first detection of what may be the ENA of heliospheric origin is due to CELIAS/HSTOF (Hilchenbach et al. 1998). The acronym denotes the High-energy Suprathermal Time-of-Flight sensor (HSTOF) of the Charge, Element and Isotope Analysis System (CELIAS). It is operating on board of the SOHO spacecraft, situated near the Lagrangian point L1 between Earth and the Sun. The instrument has the line-of-sight directed $37^{\circ}$ west off the Sun, with the field-of-view $\pm 2^{\circ}$ wide in longitude and $\pm 17^{\circ}$ in latitude. During the course of one year it scans over all directions in the ecliptic plane.

The observations of ENA are possible only during the periods of relatively low ion flux intensity, the "quiet times". For these periods, the mass $=1$ events in the low energy channels $(58-88 \mathrm{keV})$ were interpreted as neutral hydrogen atoms. The results are shown in Fig. 12. These include the changes due to in-flight new calibration of the instrument (Hilchenbach et al. 2000) which raised the previous estimations of the flux by a factor of 10. Note that there are many data points for the two first years of observations (1996 and 1997) which were characterized by low solar activity. The quiet time mass $=1$ flux in the 55-80 keV range peaked during the periods near $\mathrm{DOY}=200$, close to the time $(\mathrm{DOY}=194)$ when the instrument's line-of-sight was directed towards anti-apex of the LISM. In 1998 the contact with SOHO was lost for a period of time including DOY 200. The high solar activity during 1999 reduced the quiet time periods, and in 2000 the situation was even worse due to a big solar flare ("Bastille Day flare" of June 14th).

The observations are compared with the calculated ENA hydrogen flux from the pick-up proton distribution for the $\alpha=-3$ case, at $63 \mathrm{keV}$ (which approximates the average energy of the observed particles in the $58-88 \mathrm{keV}$ range). With no adjustments in the model parameters, the calculated flux intensity is close to the observed flux. Note a small shift of the observed flux peaks from the anti-apex position. This is not necessarily inconsistent with the PUI ENA model, because the direction towards the heliotail may be different from the anti-apex due to the interstellar magnetic field (Fahr et al. 1988; Ratkiewicz et al. 1998; Czechowski \& Grzedzielski 1998).

Figures 13 and 14 present the results for the ENA energy spectrum. To derive the spectrum from the SOHO data one must subtract the flux of protons which penetrate into the instrument from the measured total mass $=1$ flux. The uncertainty in the quiet time proton flux (assumed to follow a power law of $E^{-2.5}$ ) and in the probability of 
proton transmission would introduce a systematic error in the ENA flux. Only for the three lowest energy data points this error is expected to be small $(<10 \%$, Hilchenbach et al. 2000). The calculated spectrum is clearly steeper than the data.

\section{Conclusions}

We have considered a model of the evolution of the pickup ion distribution including both the pre-shock and the post-shock regions of the heliosphere. The pre-acceleration of the pick-up ions by the turbulence in the pre-shock solar wind was taken into account. The results suggest that the energetic ion spectrum near the termination shock may, for the energies below $50-100 \mathrm{keV}$, be determined by the shock-modified pre-accelerated pick-up ion spectrum rather than the power-law spectrum expected from the shock acceleration.

The ion flux at the shock obtained in the model is at low energy (up to $60-70 \mathrm{keV}$ ) significantly higher than the extrapolated ACR power law spectrum. In result, also the ENA flux from the pick-up ions is high and agrees with the observations by CELIAS/HSTOF (the alternative interpretation, assuming that the ENA come from the shock-accelerated ACR particles, may have problems with explaining the observed ENA flux level: see Czechowski et al. 2001). The directional dependence of the ENA flux is also in agreement with observations, with the flux intensity peaks from the (approximately) anti-apex direction. This may be an indication that the ENA flux comes from the post-shock region, although there is a possible alternative (Kota et al. 2001) connected with the CIR accelerated ions interacting by charge-exchange with the helium atoms in the region where the helium density is increased due to gravitational focusing (the helium cone). Although the errors are large, the data suggest the anti-apex to apex flux ratio lower than calculated. This may imply that the model of the heliosphere used in the calculations (Fahr et al. 2000; Czechowski et al. 2001) should be modified. Another possibility is that a part of the ENA flux comes from another source, with no peaks from the anti-apex direction.

The results are consistent with the "normal turbulence" case, with the amplitude of the magnetic field perturbations evolving adiabatically $\left(\tilde{B}^{2} \propto r^{-3}\right.$ case). The other version of the model (persisting source of the perturbations, $\tilde{B}^{2} \propto r^{-2}$ ) does not fit the ENA data, but it is not realistic enough in any case (too large total energy in the PUI spectrum).

The calculated ENA energy spectrum is steeper than the spectrum deduced from CELIAS/HSTOF data. The experimental spectrum is, however, sensitive to assumptions about the proton energy spectrum and the transmission function. Also, the PUI model disregards the effects of diffusive shock acceleration, which can in fact become important in the considered energy range and make the proton and the ENA spectrum less steep.

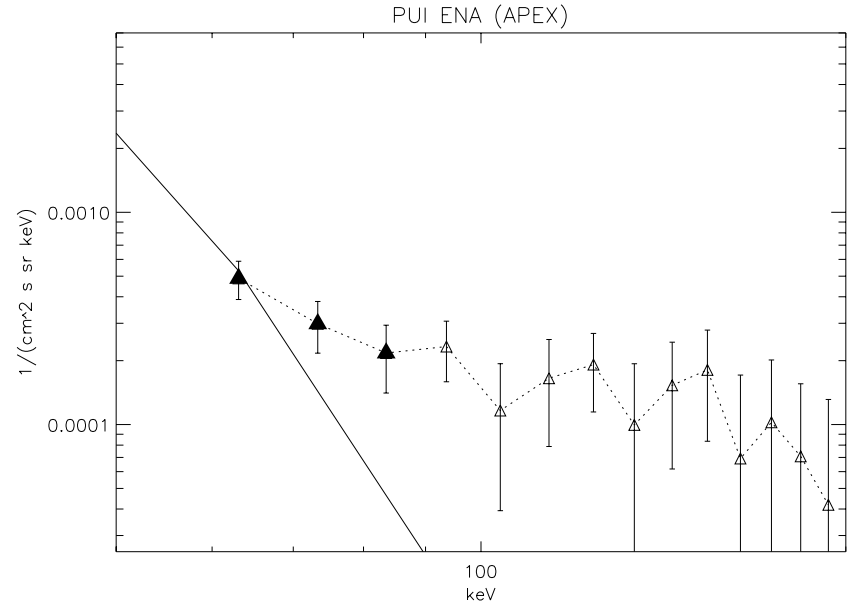

Fig. 13. CELIAS/HSTOF data compared with the PUI ENA hydrogen energy spectrum (flux from the forward direction, $\alpha=-3)$. Only the first three low energy data points (marked by filled triangles) are expected to represent the neutral flux (see the text). The data points above $90 \mathrm{keV}$ (open triangles) depend significantly on the quiet time proton flux. The background (not shown) is of the order of $1-2 \times 10^{-4}\left(\mathrm{~cm}^{2} \text { s sr keV }\right)^{-1}$.

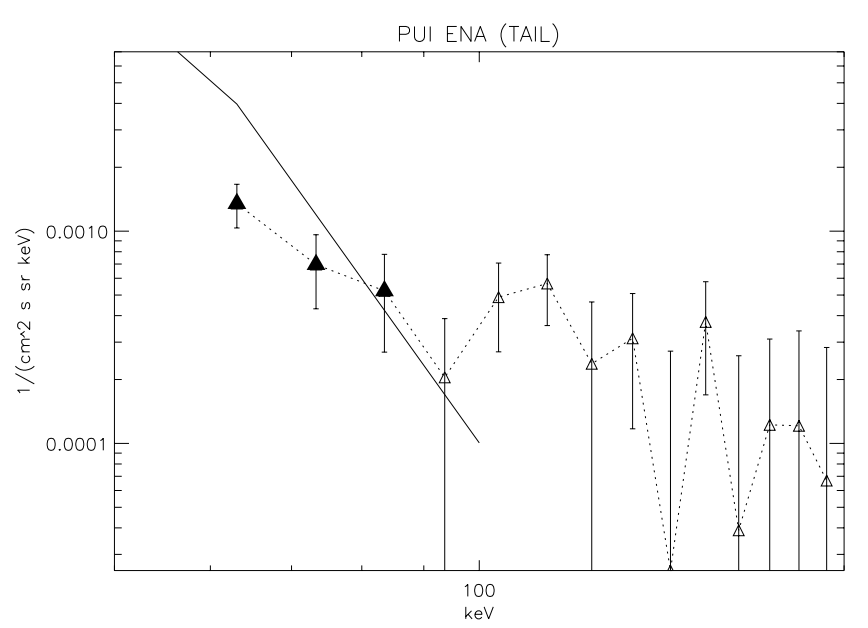

Fig. 14. As Fig. 13, but for the heliotail direction.

The energy range of the CELIAS/HSTOF observations $(58-88 \mathrm{keV})$ coincides with the region where the PUI and PUI ENA spectrum decrease very fast (for the normal turbulence case) and other contributions may appear. The forthcoming data from INCA/Cassini (Krimigis et al. 2000) and HENA/Image (Mitchell et al. 2000; first results: Roelof 2000) which should include also the lower energy region where the model predicts high ENA flux, are therefore of particular interest.

Acknowledgements. A.C. is grateful for financial support within the framework of a Polish-German cooperation (project 436 POL 113/80/0) and acknowledges support from KBN grant 8 T12E 029 20. A.C. also wishes to thank the MaxPlanck-Institut für Aeronomie for hospitality. 


\section{References}

Baranov, V. B., \& Malama, Yu. G. 1993, J. Geophys. Res., 98, 15157

Barnett, C. F., Hunter, H. T., Kirkpatrick, M. I., \& Phaneuf, R. A. 1990, Atomic Data for Fusion. Collisions of H, H2, $\mathrm{He}$ and Li Atoms and Ions with Atoms and Molecules, Oak Ridge Natl. Lab. Report, ORNL-6086-VI, Oak Ridge, Tenn. (1990)

Bogdan, T. J., Lee, M. A., \& Schneider, P. 1991, J. Geophys. Res., 96, 161

Chalov, S. V., \& Fahr, H. J. 1994, A\&A, 288, 973

Chalov, S. V., \& Fahr, H. J. 1995, Planet. Space Sci., 43, 1035

Chalov, S. V., \& Fahr, H. J. 1996, Solar Phys., 168, 389

Chalov, S. V., \& Fahr, H. J. 1997, A\&A, 326, 860

Chalov, S. V., \& Fahr, H. J. 2000, A\&A, 360, 381

Chalov, S. V., Fahr, H. J., \& Izmodenov, V. 1995, A\&A, 304, 609

Chalov, S. V., Fahr, H. J., \& Izmodenov, V. 1997, A\&A, 320, 659

Czechowski, A., Grzedzielski, S., \& Mostafa, I. 1995, A\&A, 297,892

Czechowski, A., \& Grzedzielski, S. 1998, Geophys. Res. Lett., 25,1855

Czechowski, A., Fichtner, H., Grzedzielski, S., et al. 1999a, Proceedings of the 26th International Cosmic Ray Conference, Salt Lake City, Utah, August 17-25 1999, ed. D. Kieda, M. Salamon \& B. Dingus, vol. 7, 589

Czechowski, A., Fahr, H. J., Fichtner, H., \& Kausch, T. 1999b, Proceedings of the 26th International Cosmic Ray Conference, Salt Lake City, Utah, August 17-25 1999, ed. D. Kieda, M. Salamon, \& B. Dingus, vol. 7, 465

Czechowski, A., Fichtner, H., \& Kausch, T. 1999c, Proceedings of the 26th International Cosmic Ray Conference, Salt Lake City, Utah, August 17-25 1999, ed. D. Kieda, M. Salamon and B. Dingus, vol. 7, 523

Czechowski, A., Fichtner, H., Grzedzielski, S., et al. 2001, A\&A, 368, 622

Donohue, D. J., \& Zank, G. P. 1993, J. Geophys. Res., 98, 19005

Dworsky, A., \& Fahr, H. J. 2000, A\&A, 353, L1

Fahr, H. J., Grzedzielski, S., \& Ratkiewicz, R. 1988, Ann. Geophys., 6, 337

Fahr, H. J., Kausch, T., \& Scherer, H. 2000, A\&A, 357, 268

Fahr, H. J., \& Lay, G. 2000, A\&A, 356, 327

Fahr, H. J., \& Rucinski, D. 1999, A\&A, 350, 1071

Giacalone, J. J., \& Jokipii, J. R. 1998, Space Sci. Rev., 83, 282
Hilchenbach, M., Hsieh, K. C., Hovestadt, D., et al. 1998, ApJ, 503, 916

Hilchenbach, M., Hsieh, K. C., Hovestadt, D., et al. 2000, COSPAR Colloquium on: The Outer Heliosphere: The Next Frontiers, Potsdam, Germany, 24-28 July 2000

Hsieh, K. C., Shih, K. L., Jokipii, J. R., \& Grzedzielski, S. 1992, ApJ, 393, 756

Isenberg, P. A. 1987, J. Geophys. Res., 92, 1067

Kausch, T., \& Fahr, H. J. 1997, A\&A, 325, 828

Kausch, T. 1998, Ph.D. Thesis, University of Bonn

Kota, J., Hsieh, K. C., Czechowski, A., et al. 2001, J. Geophys. Res., in press

Krimigis, S. K., et al. 2000, Space Sci. Rev., in press

Kucharek, H., \& Scholer, M. 1995, J. Geophys. Res., 100, 1745

Lee, M. A. 1983, J. Geophys. Res., 88, 6109

Lee, M. A., Shapiro, V. D., \& Sagdeev, R. Z. 1996 J. Geophys. Res., 101, 4777

Le Roux, J. A., \& Fichtner, H. 1997, J. Geophys. Res., 102, 17365

Le Roux, J. A., Potgieter, M. S., \& Ptuskin, V. S. 1996, J. Geophys. Res., 101, 4791

Le Roux, J. A., Fichtner, H., Zank, G. P., \& Ptuskin, V. S. 2000, J. Geophys. Res., 105, 12557

Matthaeus, W. H., Zank, G. P., Smith, C. W., \& Oughton, S. 1999, Phys. Rev. Lett., 82, 3444

Mitchell, D. G., Jaskulek, S., Schlemm, C. E., et al. 2000, Space Sci. Rev., 91, 1

Parker, E. N. 1965, Planet. Space Sci., 13, 9

Potgieter, M. S., \& Moraal, H. 1988, ApJ, 330, 445

Ratkiewicz, R., Barnes, A., Molvik, G. A., et al. 1998, A\&A, 335,363

Roelof, E. C. 2000, COSPAR Colloquium on: The Outer Heliosphere: The New Frontiers, Potsdam, Germany, 2428 July 2000

Rucinski, D., Fahr, H. J., \& Grzedzielski, S. 1993, Planet. Space Sci., 41, 773

Scholer, M., \& Kucharek, H. 1999, Geophys. Res. Lett., 26, 29

Stone, E. C., Cummings, A. C., \& Webber, W. R. 1996, J. Geophys. Res., 101, 11017

Zank, G. P., Webb, G. M., \& Donohue, D. J. 1993, ApJ, 404, 67

Zank, G. P., Matthaeus, W. H., \& Smith, C. W. 1996a, J. Geophys. Res., 101, 17093

Zank, G. P., Pauls, H. L., Cairns, I. H., \& Webb, G. M. 1996b, J. Geophys. Res., 101, 457

Zank, G. P. 1999, Space Sci. Rev., 89, 413

Zank, G. P., \& Cairns, I. H. 2000, ApJ, 541, 489 